\title{
Increasing influenza vaccination coverage in healthcare workers: a review on campaign strategies and their effect
}

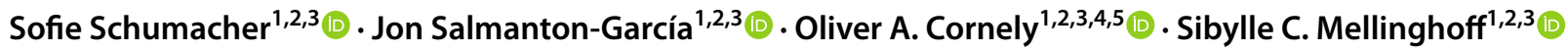

Received: 1 October 2020 / Accepted: 6 November 2020 / Published online: 7 December 2020

(c) The Author(s) 2020

\begin{abstract}
Purpose Increasing influenza vaccination coverage in healthcare workers is a challenge. Especially during the ongoing COVID-19 pandemic, high vaccination coverage should be attained. This review analyzed strategies to increase influenza vaccination coverage in healthcare workers.

Methods A literature search using PubMed was conducted and 32 publications on influenza vaccination campaigns for healthcare workers were reviewed for key interventions and resulting vaccination coverage.

Results Among key interventions analyzed, mandatory vaccination policies or multifaceted campaigns including a vaccinateor-wear-a-mask policy as well as mandatory declination reached vaccination coverage in healthcare workers of over $90 \%$. Although campaigns solely based on education and promotion or on-site-vaccination did not regularly exceed an absolute vaccination coverage of $40 \%$, a substantial relative increase in vaccination coverage was reached by implementation of these strategies.

Conclusion Mandatory vaccination policies are effective measures to achieve high overall vaccination coverage. In clinics where policies are infeasible, multifaceted campaigns comprising on-site vaccination, vaccination stands and educational and promotional campaigns as well as incentives should be implemented. Lessons learned from influenza campaigns could be implemented in future SARS-CoV-2 vaccination campaigns.
\end{abstract}

Keywords Healthcare personnel $\cdot$ Seasonal influenza $\cdot$ Influenza virus $\cdot$ Immunization $\cdot$ Vaccine uptake rate $\cdot$ Immunization programs

Oliver A. Cornely and Sibylle C. Mellinghoff are contributing equally to this work.

Oliver A. Cornely

Oliver.Cornely@uk-koeln.de

1 Faculty of Medicine and University Hospital Cologne, Department I of Internal Medicine, Excellence Center for Medical Mycology (ECMM), University of Cologne, Herderstraße 52-54, 50931 Cologne, Germany

2 Faculty of Medicine and University Hospital Cologne, Cologne Excellence Cluster On Cellular Stress Responses in Aging-Associated Diseases (CECAD), Chair Translational Research, University of Cologne, Cologne, Germany

3 German Centre for Infection Research (DZIF), Partner Site Bonn-Cologne, Cologne, Germany

4 Faculty of Medicine and University Hospital Cologne, Clinical Trials Centre Cologne (ZKS Köln), University of Cologne, Cologne, Germany

5 Center for Molecular Medicine Cologne (CMMC), University of Cologne, Cologne, Germany

\section{Introduction}

Influenza is a highly contagious disease, causing 4.0-8.8 respiratory deaths per 100000 individuals annually worldwide [1]. Vaccination is the most effective form of influenza prevention. Children under 5 years of age, chronically ill and immunocompromised patients, the elderly ( $>65$ years) and pregnant women are at high risk of complicated influenza courses. The World Health Organization (WHO) recommends annual influenza vaccination for these vulnerable populations as well as healthcare workers (HCW) [2]. HCW may transmit influenza to vulnerable patients, thereby compromising patient safety [3].

Despite this recommendation, vaccination rates among $\mathrm{HCW}$ are low ranging from 15.6 to $63.2 \%$ (median 30.2\%) in Europe [4]. Other than allergies against vaccine compounds, there are no medical contraindications for influenza vaccination. If allergy to egg protein is known, a cell- or recombinant-based vaccine can be used [5]. The challenge 
is addressing personal reasons among unvaccinated staff against influenza vaccination. In the German OKaPII study, doctors stated mainly organizational aspects, whereas nurses declared lacking confidence in efficacy and safety of vaccines [6]. Organizational and educational issues can be approached and overcome. It should, therefore, be possible to increase influenza vaccination rates.

Most university hospitals in Germany treat high numbers of vulnerable patients. As immunocompromised patients may have an impaired immune response to vaccines, herd immunity is even more important [7]. To protect these patients, high influenza vaccination rates in $\mathrm{HCW}$ have to be achieved [8]. At the University Hospital of Cologne, we are planning an intensified influenza vaccination campaign for the upcoming season 2020/2021. Therefore, we analyzed the current literature on influenza vaccination campaigns for $\mathrm{HCW}$.

In context of the ongoing coronavirus disease 2019 (COVID-19) pandemic, public health implications of the influenza season 2020/2021 must be considered. Coinfections of severe acute respiratory syndrome coronavirus 2 (SARS-CoV-2) and influenza virus have been described [9]. Sick leaves of HCW due to influenza or coinfections with SARS-CoV-2 could impact workforce availability. This, in combination with high infection rates in patients, could overburden our healthcare systems. Thus, high influenza vaccination rates among HCW should be attained [10].

\section{Methods}

To identify influenza vaccination campaign strategies, we performed a literature search using the PubMed® database. The following query was defined: (("health personnel/analysis" [MeSH Terms] OR "health personnel/statistics and numerical data" [MeSH Terms]) AND "influenza, human/ prevention and control" [MeSH Terms]) NOT (review [Publication Type]). Articles published from January 2010 to August 2020 were included. No language restrictions were applied. Publications were selected by screening title and abstract. Studies implementing interventions to increase seasonal influenza vaccination rates among $\mathrm{HCW}$ were included. Studies focusing on pandemic influenza in 2009-2010 were excluded. The interventions needed to be clearly defined. Also, the selected studies had to include an evaluation of effect in comparison to a control group or in comparison to at least one previous season. Studies which conducted surveys in a number of institutions comparing different campaign strategies among each other were excluded. If the studies differentiated between nursing homes and acute care hospitals, we focused on acute care hospitals. Additionally, references of relevant publications were examined to identify further suitable studies (Fig. 1).
Each publication was reviewed for key interventions and resulting vaccination coverage (VC). $\mathrm{VC}$ was defined as the proportion of the vaccinated population in relation to the entire study population. The interventions of interest were education and promotion, incentives, organization, and policies. Education and promotion included providing material and spreading awareness. Incentives included free vaccine or giving away prizes among the vaccinated. Organizational interventions contained on-site vaccination, peer-to-peer vaccination, mass vaccination events and assignment of dedicated staff. Mandatory vaccination, vaccinate-or-weara-mask policies and declination forms to be submitted by unvaccinated HCW were grouped under policies. Moreover, combinations of these interventions were examined. The effect of the implemented strategy was evaluated by comparing $\mathrm{VC}$ before and after intervention. The relative increase in percent between initial and resulting $\mathrm{VC}$ was considered to evaluate the potential increase in $\mathrm{VC}$ regarding the key interventions.

\section{Results}

\section{Literature search}

Our initial search yielded 231 publications. After screening titles and abstracts, 41 publications remained. These fulltext articles were assessed and subsequently 23 studies were included. Additionally, 9 publications found through references of relevant literature were added. In total, 32 articles were reviewed (Fig. 1).

Of the selected studies, 14 were conducted in the USA, 5 in Italy, 3 in Australia, and 1 each in Canada, France, Germany, Israel, Japan, Korea, Qatar, Spain, Switzerland, and Turkey. Most $(n=28)$ studies compared VC before and after a specific vaccination campaign conducted at individual or clustered institutions. Other studies $(n=4)$ compared vaccination rates between an intervention group and a control group. The majority $(n=30)$ of the studies were performed in hospitals, while two studies only analyzed nursing homes. In the following sections, the outcome per key interventions is described. Further details such as number of subjects described in each study can be found in Table 1. One study is listed under two key interventions [11].

\section{Key intervention: education and promotion}

Among the selected studies, six built their campaign mainly upon educational and promotional aspects [12-17]. Overall, the key intervention education and promotion increased VC relatively by $65.9 \%$ (standard deviation (SD): $\pm 55.8 \%$, range: $14.5-162.5 \%$ ) (Table1). 


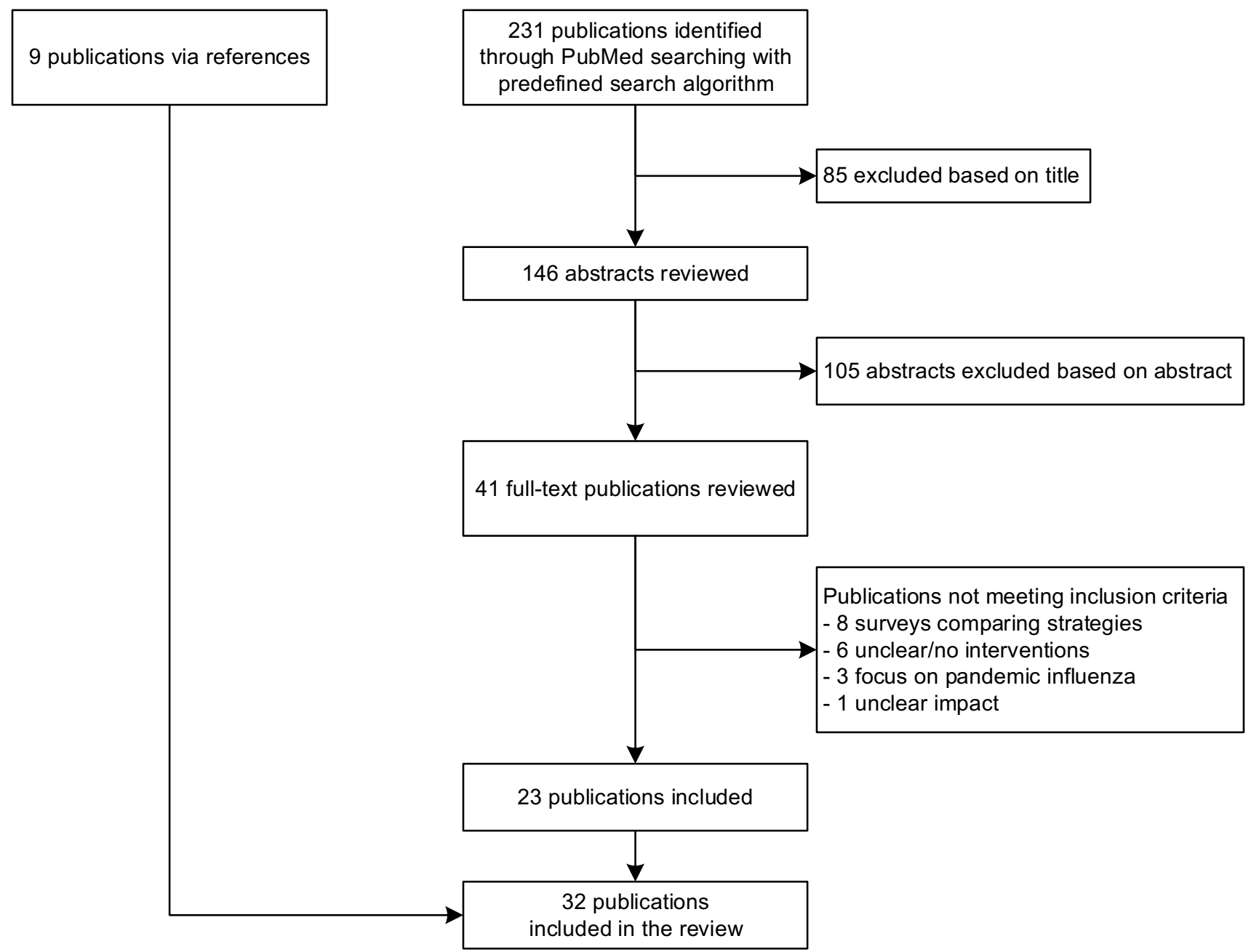

Fig. 1 Study selection flow. Flowchart showing the study selection after searching with the following predefined search algorithm on PubMed®: [("health personnel/analysis" (MeSH Terms) OR "health personnel/statistics and numerical data" (MeSH Terms)] AND "influ-

In one randomized trial from Israel, the intervention group $(n=163)$ received a lecture session, recurring emails containing literature as well as reminders and an appointed key figure from each department personally talked to each participant of the intervention group. Compared to the initial $\mathrm{VC}$ of $27 \%$, the final $\mathrm{VC}$ was $53 \%$ in the intervention group. The VC in the control group increased from 20 to $27 \%$ [12]. A cluster-randomized controlled trial conducted in French nursing homes included slideshows and posters regarding prejudices against and reasons for influenza vaccination. VC increased from 28 to $34 \%$ in the intervention group. VC decreased from 24 to $23 \%$ in the control group [13]. A 1-h training course for all participants concerning influenza vaccination guidelines, vaccine types and administration was used as an intervention in an Italian study. Subsequently, the initial VC of $16 \%$ increased to a final VC of $42 \%$ in the intervention group. In comparison, $\mathrm{VC}$ increased from 13 enza, human/prevention and control" (MeSH Terms)] NOT [review (Publication Type)]. Additionally, nine publications were found through references of relevant publications

to $31 \%$ in the control group [14]. A different Italian hospital appealed on personal as well as patient safety. It comprised posters in frequented areas, distribution of factsheets and intranet presence. Most survey participants (66\%) agreed that the information was useful. Following the implementation of the toolkit, vaccination coverage was $14 \%$ which corresponded to earlier VC of $10 \%$ [15]. During a Korean campaign, unvaccinated $\mathrm{HCW}$ were contacted via phone for a ten-minute educational presentation. As this had no effect, unvaccinated medical doctors then received one-onone educational counseling with on-site vaccination (OSV). VC increased from 83 to 93\% [16]. In a Spanish before-andafter-trial, the key intervention consisted of a "I've already been vaccinated" webpage showing humorous pictures of all heads of departments as well as a vaccinated pregnant woman promoting vaccination also during pregnancy. The 


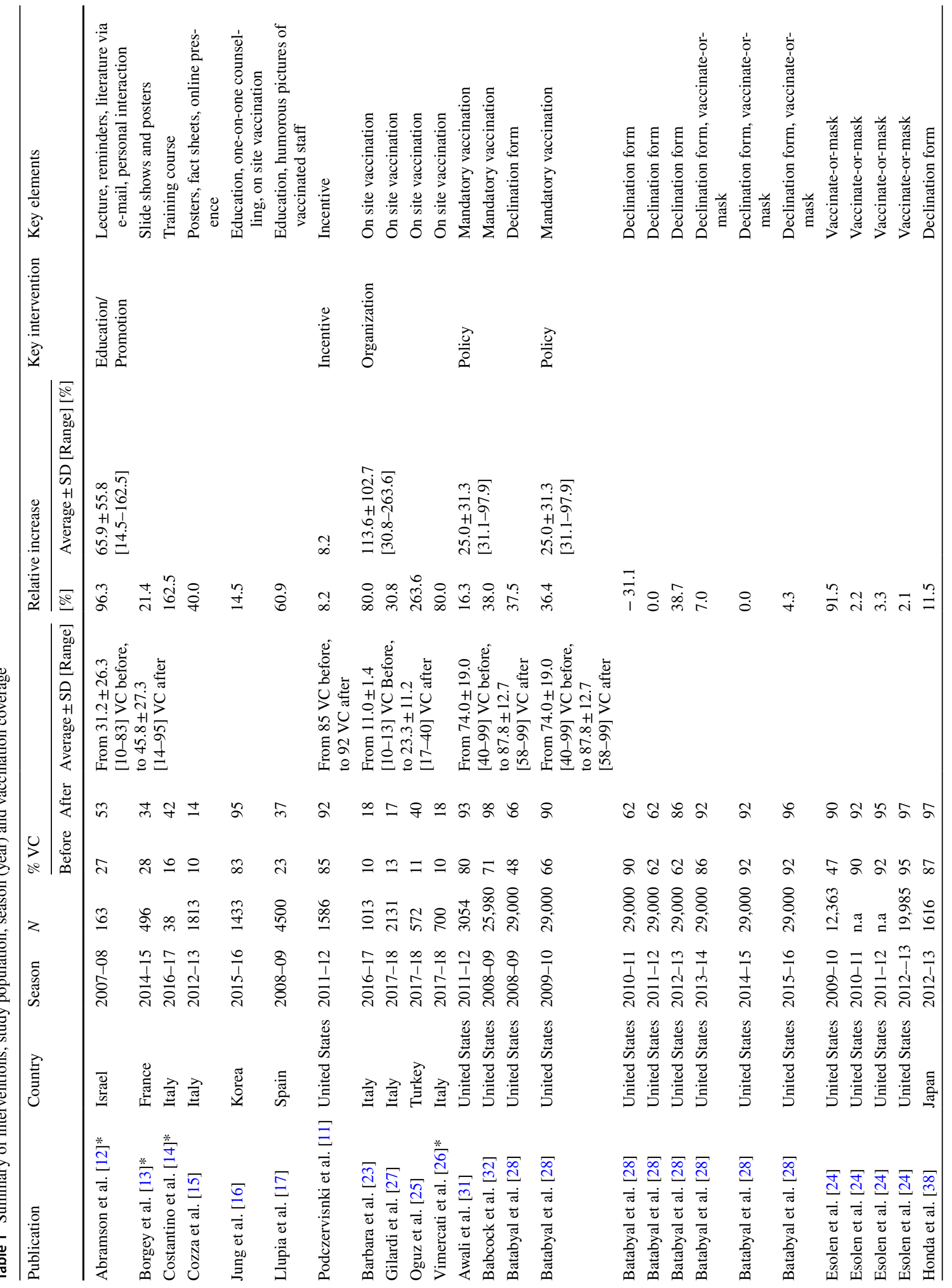




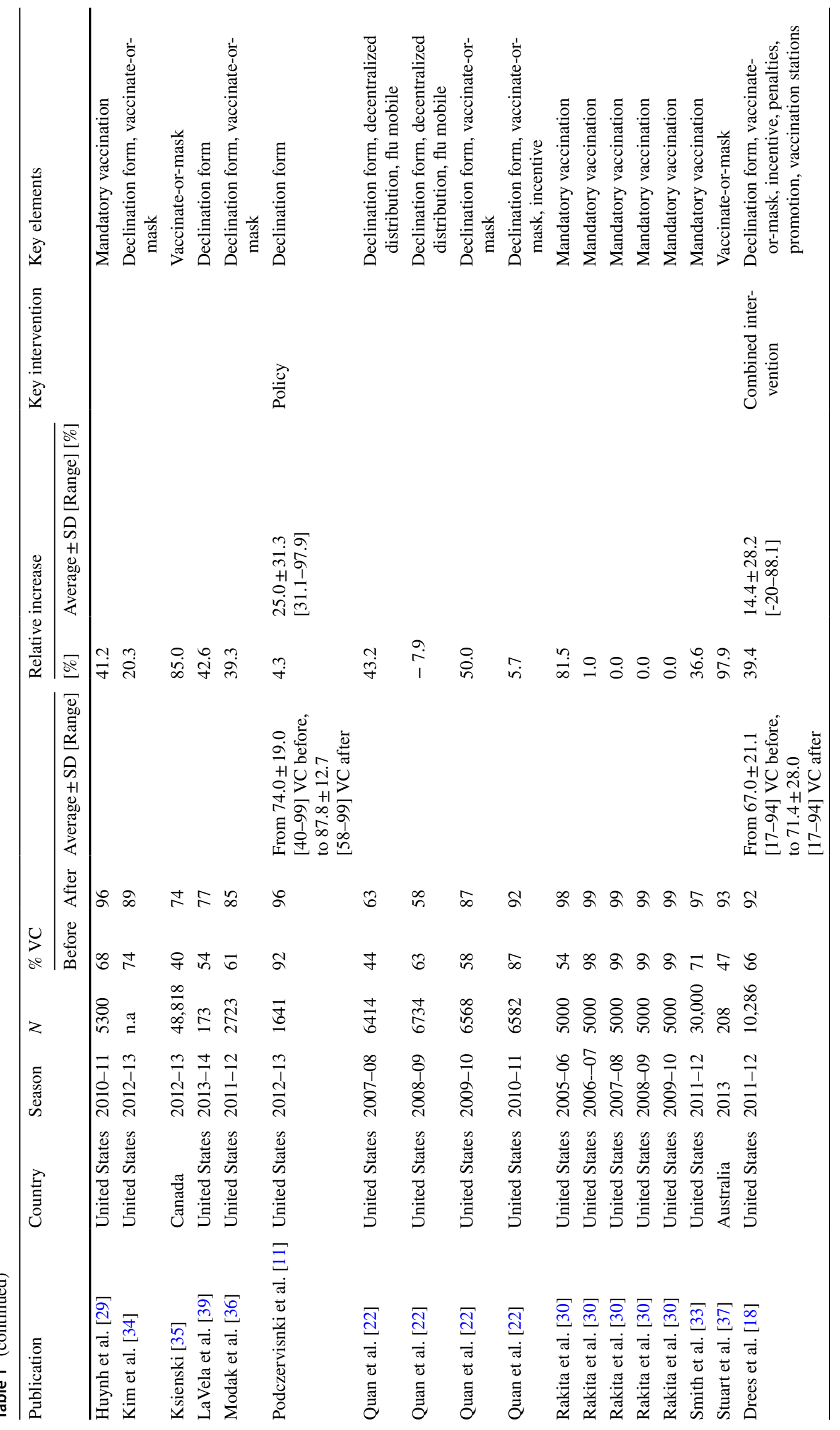




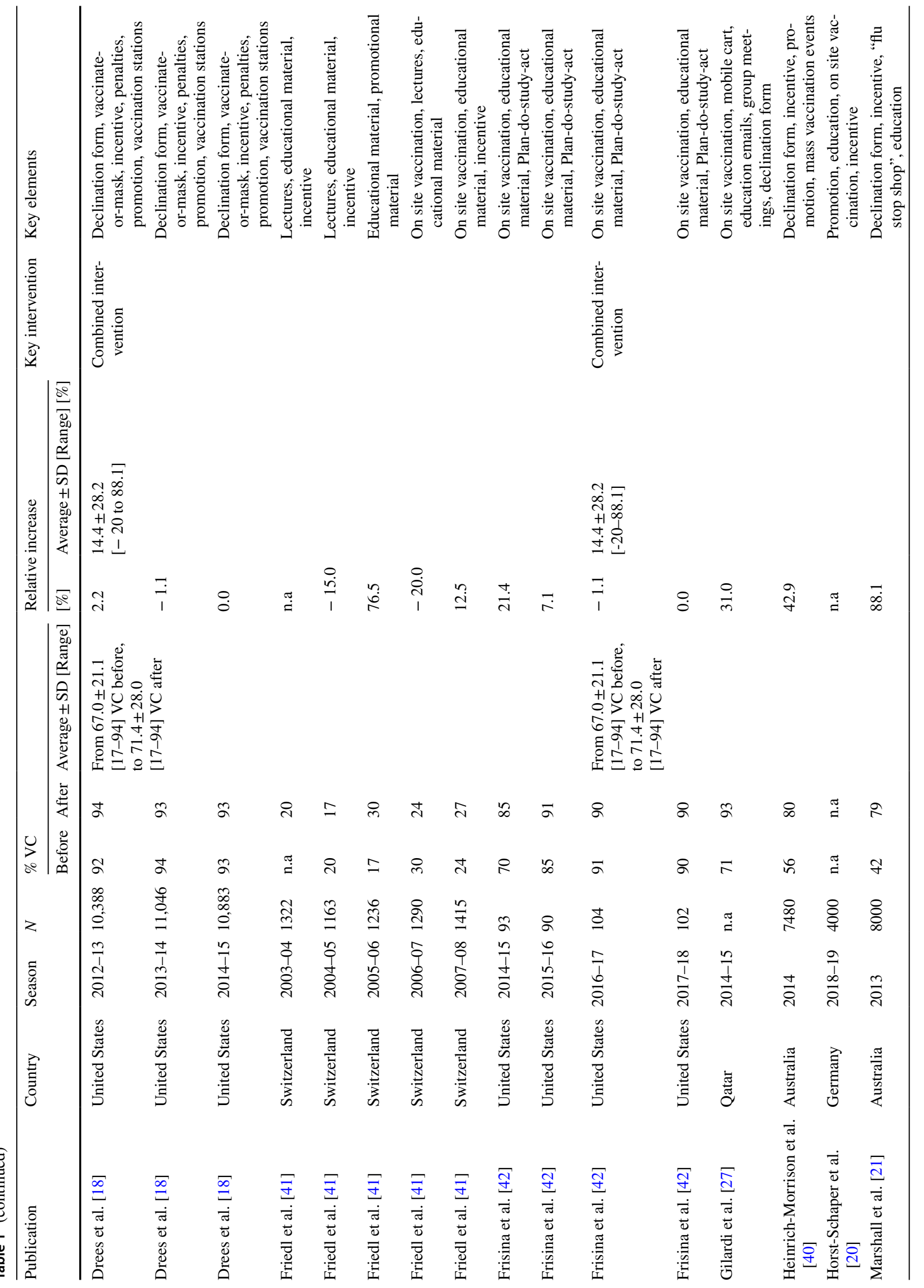




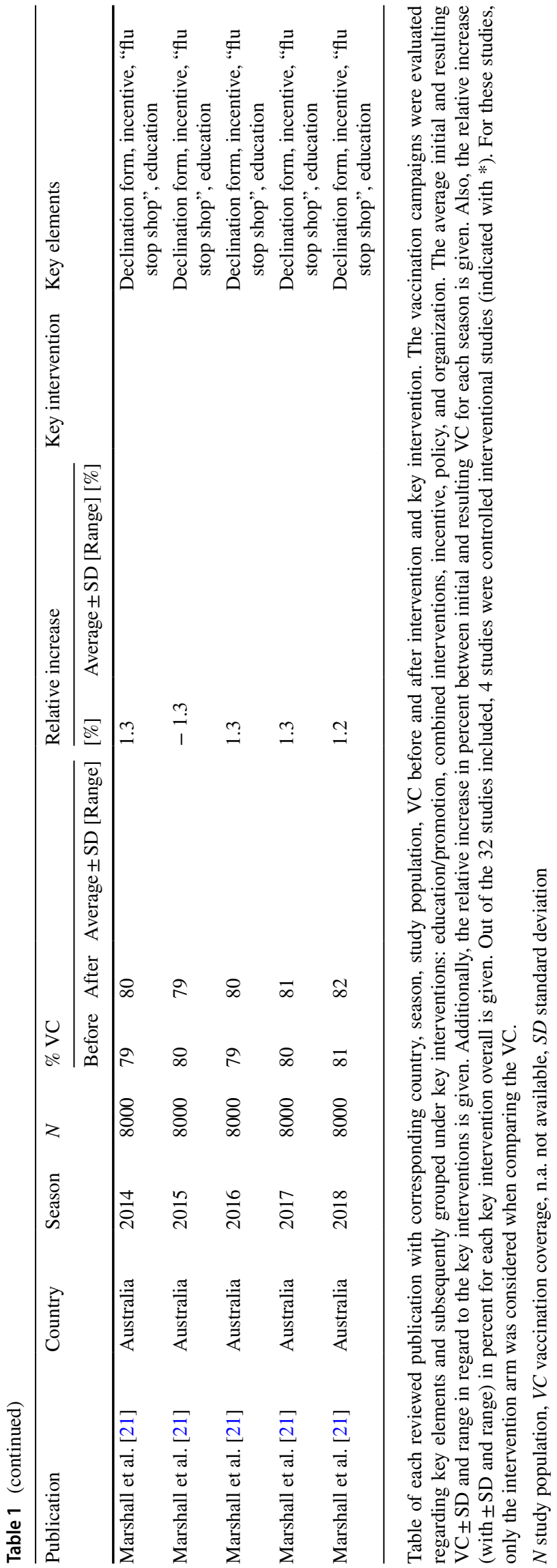

authors concluded that the campaign encouraged the discourse on vaccination increasing VC from 23 to $37 \%$ [17].

\section{Key intervention: incentives}

Incentives were emphasized as a key intervention in one study. In six other studies, incentives were used as part of multifaceted campaigns [11, 17-22].

The above-mentioned study provided a 25 US Dollar gift card for every employee, if the overall VC reached $95 \%$. This approach increased the VC from 87 to $92 \%$ [11]. The key intervention incentive increased VC relatively by $8.2 \%$ (Table1).

An employee-bonus program was implemented in two studies $[18,19]$. A prize draw among vaccinated staff was part of two campaigns $[17,20]$. In one multifaceted campaign, prizes where given to wards if the target $\mathrm{VC}$ was achieved [21]. One study took a different approach creating a disincentive for department leaders. Departments could lose budget allocations if vaccination rates were unsatisfactory. This increased vaccination rates from 87 to $92 \%$ [22].

The vaccine was offered free of charge in the respective prior season and during all included campaigns. Therefore, no aspect in this regard can be reported.

\section{Key intervention: organizational strategies}

Organizational aspects which facilitated access to the vaccine were implemented in eight studies [18, 21-27]; however, OSV was highlighted as a main intervention in only four campaigns [23, 25-27]. OSV as implemented key intervention increased VC overall by $113.6 \%$ (SD: $\pm 102.7 \%$, range 30.8\%-263.6\%) (Table1). An Italian teaching hospital introduced OSV observing an increase in vaccination rates in medical residents from 10 to $18 \%$ [23]. In a different Italian study, the VC increased from 10 to $18 \%$ in the intervention group after offering OSV. Of note, out of the vaccinated $\mathrm{HCW}, 80 \%$ received vaccination on-site. In comparison, VC increased by $1.5 \%$ in the control group (without offered OSV). Initial and resulting overall VC was not provided by the authors for the control arm [26]. At another Italian hospital, a promotional campaign as well as OSV had already been in place in previous seasons with $\mathrm{VC}$ of $13 \%$. Increased availability of the vaccine through extended OSV as well as longer timeslots at vaccination stations and at the occupational health department were added increasing VC to $17 \%$ [27]. After offering OSV, the VC increased from 11 to $40 \%$ in a Turkish children's Hospital [25].

The following studies used special organizational strategies as part of their campaigns and are discussed under their respective subheading. An approach using peer-topeer vaccination was taken by two hospitals $[22,24]$. A flu kit including the vaccine, consent forms and stickers was 
handed out to appointed team leaders of individual departments [24]. In the second clinic nurse managers could receive vaccines from the Occupational Health Department to distribute among their personnel [22]. A "flu-stop-shop" in a main area was organized in one Australian study. During the campaign, $\mathrm{HCW}$ could receive vaccination at the "flu-stop-shop" at all times without appointment [21]. In another study, a "blitz" campaign was conducted during the first 2 weeks of October. Vaccination stations were set up at all entrances of the hospital. Consequently, about $70 \%$ of all employees were vaccinated in the first 2 weeks [18].

\section{Key intervention: policies}

Among the selected studies, 15 included policies as key interventions. Overall, policies increased VC relatively by $25.0 \%$ (SD: $\pm 31.3 \%$, range $31.1 \%-97.9 \%$ ) (Table1). One study conducted in the USA analyzed the effect of several different policies from 2008 to 2016. During seasons, in which policies included a signed declination option, the VC varied from 62 to $66 \%$. Upon addition of educational aspects, VC increased to $86 \%$. After a state-wide mandate in 2013 , requiring unvaccinated staff to wear a mask, a maintained VC of $92-96 \%$ over the course of three seasons was reached [28].

In five studies, influenza vaccination was mandatory for HCW [29-33]. These publications were exclusively from the USA. Before implementation of the mandate, multifaceted vaccination campaigns had already been in place in all five studies with $\mathrm{VC}$ ranging from 54 to $80 \%$. After influenza vaccination was made an employment requirement VC was 93-98\%. In every study, "mandatory" implied that contracts with unvaccinated staff without exemptions were to be terminated. Overall, none to $0.14 \%$ of staff contracts were terminated due to the mandate. All five campaigns granted medical or religious exemptions. Egg allergy, history of Guillain-Barré syndrome and previously reported severe vaccine reaction were among the regarded exemptions. Exemptions due to medical reasons were acknowledged to $0.7-1.9 \%$ of staff and religious exemptions to $0.13-0.3 \%$. One study declared that exemption requests reflected misinformation regarding the vaccine. These exemption requests included immunosuppression or pregnancy as reasons, although vaccination is recommended for both of these conditions [32]. Except for one hospital [29], the exempted unvaccinated staff had to wear a mask during influenza season.

A vaccinate-or-wear-a-mask approach was a key intervention in six publications [22, 24, 34-37]. A deadline for vaccination was set, after which unvaccinated staff had to wear a mask for the duration of the influenza season [34-36]. Vaccinated staff partially had markings on identification badges $[22,35,36]$. Supervisors were informed of their employees' vaccination status and were held accountable in three campaigns $[22,24,36]$. One study implemented a 100 US Dollar fine for noncompliant staff [34]. Another study initially implemented contract termination as consequence of noncompliance, but was forced to retract due to litigation [35]. A sustained VC of $90-97 \%$ over 4 years, was achieved through a vaccinate-or-wear-a-mask policy in combination with a decentralized vaccine supply (complete vaccine kits for appointed team captains of different departments) in one study [24]. An Australian pilot study applied a vaccinate-orwear-a-mask mandate in the nephrology department increasing VC from 47 to $93 \%(n=208)$ [37]. Amid the six studies, three also included a declination form [22, 34, 36]. Overall, remarkable increases up to $97 \%$ in VC were observed after mask mandate [22, 24, 34-37].

Declination forms as a key intervention were used in three of the reviewed studies performed in Japan and the USA $[11,38,39]$. HCW refusing vaccination had to complete a declination form stating their reasons in all three studies. In a Japanese study noncompliant $\mathrm{HCW}$, who neither received vaccine nor handed in declination forms, were interviewed by the hospital vice president. After implementing the mandatory declination form in this study, VC increased from 87 to $97 \%$ [38]. A pilot study conducted in a US Veterans Affairs facility included a signed statement acknowledging the personal risks and risks to others in their declination form. This study reported VC increasing from 54 to $77 \%$ [39]. Another study evaluated the impact of declination forms. Here, HCW refusing vaccination had to complete a 30-min educational module, receive one-on-one counseling and sign an attestation statement in presence of an occupational health or infection prevention staff. In cases of noncompliance, HCW were required to meet with their managers and a disciplinary letter was included in their employee file. This penalty-based approach increased VC from 92 to 96\% [11]. Declination forms also played an important role in four multifaceted campaigns, which are discussed under the subheading "Combined interventions" [18, 19, 21, 40].

\section{Combined interventions}

The following studies are campaigns which did not focus on one key intervention but rather implemented three or more interventions as multifaceted strategies (education/promotion, incentive, organization, and policies) [18-21, 40-42]. Overall, combined interventions increased VC relatively by 14.4\% (SD: $\pm 28.2 \%$; range: -20 to $88.1 \%$ ) (Table1).

For one campaign a task force led by the Infection Prevention Department incorporating Employee Health, Pharmacy, and Nursing departments among others was created. A new policy was implemented which required employees to fill out either a consent, declination or exemption form. This included attestation of vaccination elsewhere. Vaccinated 
employees were asked to wear a badge saying "I' $m$ vaccinated because I care". If the badge wasn't worn, employees had to wear a mask, regardless of vaccination status. Noncompliance was considered in performance evaluations hindering possible promotions or raises. As a financial incentive, an employee bonus program was implemented. This multifaceted campaign increased VC from 57 to $72 \%$ (in the 3 years prior to the campaign) to $92-93 \%$ sustaining for four years [18].

Similarly, an Australian campaign consisted of multiple key interventions. For 6 months each year, a full-time influenza vaccination coordinator was employed. Appointed nurses conducted the vaccinations in aforementioned "flustop-shop". An intranet page with educational and promotional input was created. Promotions were spread via intranet, stickers and posters across the hospital. The chief executive officer sent emails and held presentations promoting the campaign. If wards achieved target rates, they received prizes. A mandatory declination form was implemented. Managers had access to the vaccination status of their employees via a database and were expected to hold their employees accountable. During the 6 active years of this campaign, $\mathrm{VC}$ was $79 \%$ to $82 \%$ compared to a $\mathrm{VC}$ ranging from 42 to $48 \%$ before [21].

One hospital implemented a new multifaceted strategy on top of OSV, a mobile cart, educational input and recurring e-mails. They added educational group meetings and a mandatory declination form. Also, progress reports on VC were sent to managers and heads of departments informing them of unvaccinated staff, yet without consequences for noncompliance. This increased VC from 71 to 93\% [19].

Another Australian study introduced a database to track vaccination status of all $\mathrm{HCW}$, identification of unvaccinated staff on ID badges, a declination form and awards for VC margins reached in wards (coffee machines in case of more than $80 \% \mathrm{VC}$ ). Following this campaign, VC increased from 56 to $80 \%$ [40].

A German hospital initiated the "Be a flu fighter" campaign, thereby managing to increase their VC by 4.5 -fold. Key interventions included promotion and education, mobile vaccination teams and prize drawings as incentives among the vaccinated staff. Through the implementation of the campaign, $\mathrm{VC}$ reached $72 \%$ in physicians and $50 \%$ in nurses. Baseline values were not reported [20].

One hospital in Switzerland reported their influenza vaccination campaign being unsuccessful. The campaign included: vaccination daily during lunchtime in the cafeteria for 2 weeks, individualized mobile vaccination appointments at wards or during meetings, a "health week", incentives such as free lottery ticket or a free lunch, educational and promotional flyers and posters, influenza vaccine logo, intranet presence including "frequently asked questions", involvement of the head nurse, personal letters to employees and recurring lectures. According to the authors, the multitude of interventions, however, did not significantly increase $\mathrm{VC}$ (increase from 20 to $27 \%$ over 5 years). Among nurses the $\mathrm{VC}$ even decreased due to fear of potential short- or longterm side effects and doubts of efficacy of the vaccine [41].

As part of a quality improvement study, several plan-do-study-act (PDSA) cycles over the course of four seasons were implemented in one US study. The campaign consisted of educational aspects such as the distribution of a fact sheet and personal discussions on vaccination with $\mathrm{HCW}$. Second, vaccine availability was increased in general and specifically for night shift staff and staff in remote clinics. Also, communicational aspects were enforced by sending out monthly emails showing current influenza epidemiology with a reminder of the availability of vaccination. Because "fear of needles" was identified as a barrier during a PDSA cycle, nasal vaccination was provided reducing this obstacle. Overall, VC increased from $70 \%$ to over $90 \%$ [42].

\section{Descriptive comparison of key interventions}

As shown in Fig. 2, key interventions such as education or promotion $(n=6)$ and organization $(n=4)$ were used as interventions in campaigns with initially low $\mathrm{VC}$ (range $15-25 \%)$. Policies $(n=15)$ combined interventions $(n=7)$ and incentives $(n=1)$ were applied in studies with initially high VC (>70\%). In studies with low initial VC, the key intervention led to an increase of the $\mathrm{VC}$ ranging from 11 to $18 \%$ for organizational interventions and $25-40 \%$ for education/promotion. In studies with high initial VC, the key intervention led to an increase of the VC from 79 to $92 \%$ for policies and from 85 to $92 \%$ for incentives. No change was observed for combined interventions. In the overall group ( $n=32$, all studies), VC increased from 71 to $87 \%$.

\section{Discussion}

The analysis shows that vaccination campaigns are generally based on multifaceted vaccination strategies. Furthermore, vaccination strategies are implemented on different levels of initial vaccination rate. Most of the published vaccination strategies resulted in an increase in vaccination rates independent of the initial vaccination rate.

When taking into consideration, the overall success based on absolute VC, the most effective campaigns were those that comprised regulatory measures. Implementation of a mandatory vaccination policy generated the highest overall VC. Other policies like vaccinate-or-wear-a-mask or mandatory declination forms represented successful alternatives to mandatory vaccination. A VC of over $90 \%$ could be attained, especially if noncompliance with policies had a consequence [11]. Multifaceted campaigns which included 

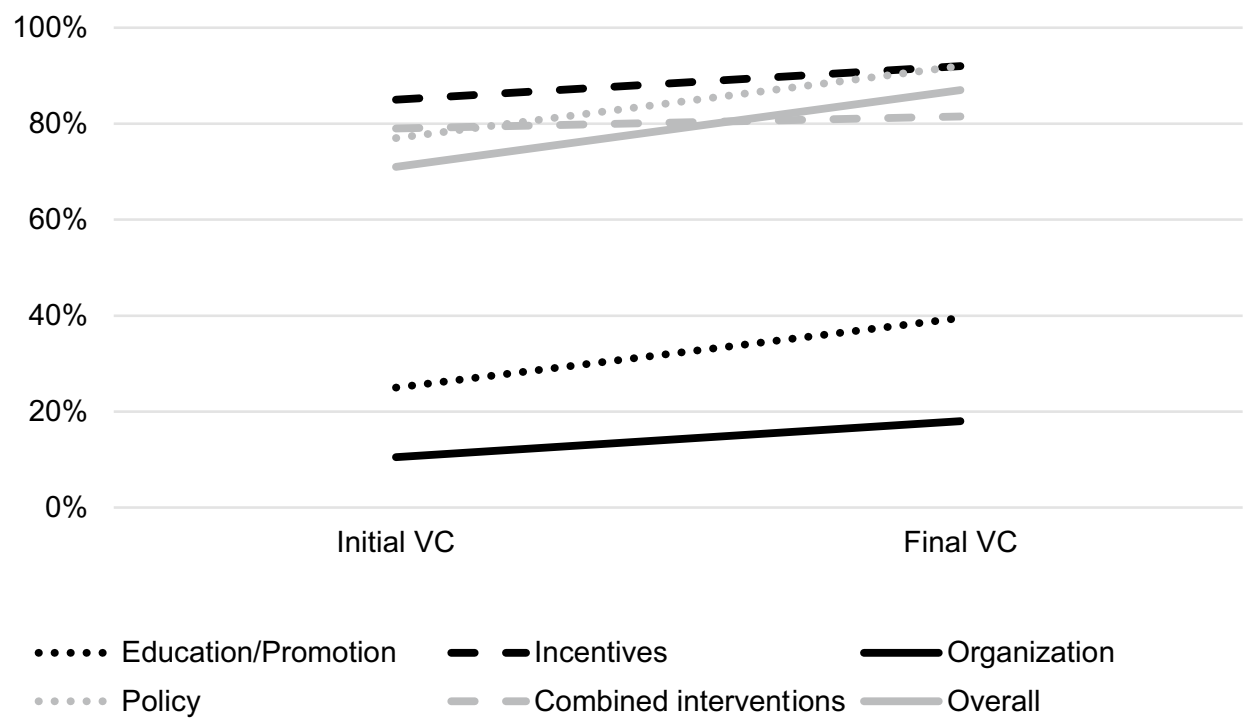

Fig. 2 Median variation of vaccination rates after the application of different policies and overall. VC, vaccination coverage. Line graph of the initial and final $\mathrm{VC}$ in regard to implemented key interventions and overall. Education/promotion $(n=6)$ included providing material and spreading awareness. Incentives $(n=1)$ included prize draws. Organization $(n=4)$ included on-site vaccination. Policies $(n=15)$

a vaccinate-or-wear-a-mask approach as well as declination forms were generally more successful than those without policies. Policies were commonly used in Asia, Australia, and the USA.

Concerning the relative increase in $\mathrm{VC}$ regardless of initial and achieved $\mathrm{VC}$, the most effective strategies were OSV (relative average increase $113.6 \%, \mathrm{SD}: \pm 102.7 \%$, range $30.8-263.6 \%$ ) and education/promotion (relative average increase $65.9 \%$, SD: $\pm 55.8 \%$, range $14.5-162.5 \%$ ). However, regarding OSV, one outlier has to be considered: after implementation of OSV in a Turkish hospital, VC increased from 11 to $40 \%$ [25]. When comparing the potential relative increase between key interventions, the heterogeneity of the studies as well as the unbalanced number of studies in regard to key interventions has to be considered. Also, the studies with the key interventions OSV as well as education/promotion did not compare longitudinal data and mostly only focused on one season. In comparison, studies which employed polices (relative average increase $25.0 \%$, SD: $\pm 31.3 \%$, range $31.1-97.9 \%$ ) and combined interventions (relative average increase $14.4 \%, \mathrm{SD}: \pm 28.2 \%$; range -20 to $88.1 \%$ ) showed an initial high increase in VC and a maintained high $\mathrm{VC}$ over the course of following seasons $[18,21,24,30,42]$. Furthermore, before the implementation of policies, multifaceted campaigns comprising educational and promotional aspects as well as OSV had already been in place in the respective studies. This shows that education and promotion as well as OSV are valuable tools to increase VC and should be implemented whenever possible. included mandatory vaccination, declination form and vaccinate-orwear-a-mask approaches. Combined interventions $(n=7)$ included combinations of the aforementioned interventions. Concerning the four controlled interventional studies, only the intervention arm was considered when comparing the VC in regard to the key intervention

However, in regard to absolute VC achieved, the key interventions education/promotion and OSV did not regularly exceed a VC of $40 \%$.

Campaigns without regulatory measures focused on organizational, educational and promotional aspects. OSV was identified as an important tool to increase VC [25]. Mass vaccination events were successful [18]. A decentralized vaccine supply using peer-to-peer vaccination was also used [22, 24]. Regarding further organizational aspects, the importance of strong leadership and representation of clinic directors and heads of departments was stressed [21, 40]. Also, the importance of a dedicated team was highlighted in almost all studies. One study explicitly recommended hiring a physician solely dedicated to the influenza campaign over the course of the season [20]. Educational and promotional aspects were used as the basis of all campaigns, but when implemented as the sole key intervention absolute VC did not exceed $40 \%$ [17]. Incentives alone were rarely used as a key intervention, but did play an important role in multifaceted campaigns. Among the studies without policies, two studies stood out regarding overall VC achieved. One attained a VC of $>90 \%$ by implementing one-on-one counseling in combination with OSV [16]. The other study conducted several PDSA cycles analyzing and addressing barriers [42]. Of note, both studies were conducted in settings with high baseline VC in Korea and the USA (Table 1). These findings are in line with other studies [43-45].

Mandatory vaccination policies are confronted with opposition and even litigations. Any form of policy 
implies tracking the vaccination status of employees. This alone is a highly controversial topic considering data protection and staff autonomy. In most European countries, mandatory vaccination policies would be hard to implement. Possibly, a vaccinate-or-wear-a-mask policy could be installed in the future considering the current COVID-19 mask policy. HCW are now sensitized on the importance of wearing a mask. A mask not only acts as an incentive to receive vaccination but also reduces influenza transmission [37]. Surprisingly, in a Swiss study, HCW partially preferred wearing a mask over receiving the vaccine [46]. Of note, a vaccinate-or-wear-a-mask approach might not be feasible as an incentive to receive the vaccine in the season 2020/2021 due to already established mask mandates in the context of the COVID-19 pandemic. Ethical implications regarding incentives like prize draws for vaccinated staff should be considered. Alternatively, incentives in an educational context like quizzes could be a way to encourage discourse on the topic. Besides these ethical considerations, monetary and human resources need to be regarded. Further research on the economic impact of $\mathrm{HCW}$ influenza vaccination on work absenteeism as well as nosocomial influenza transmission is needed. Conclusive studies could help to integrate and justify policies regarding influenza vaccination for HCW.

With the possibility of a SARS-CoV-2 vaccine, similar issues and barriers regarding $\mathrm{HCW}$ vaccination could arise. Lessons learned from influenza campaigns could help to implement successful SARS-CoV-2 vaccination campaigns in the future.

This review has limitations. First, we only searched the PubMed® database. Second, due to the heterogeneity of the studies, we had to subjectively match campaigns to key interventions. Many interventions were part of multifaceted campaigns and not studied as an individual intervention. Therefore, it is difficult to finally assess the individual impact and contribution of the key interventions. Third, we did not perform statistical analysis, but rather focused on describing the strategies and the VC individual campaigns yielded. Fourth, the used key interventions were not balanced with regards to initial VC.

In conclusion, an influenza VC of over $90 \%$ in $\mathrm{HCW}$ can be reached by mandatory vaccination policies and through multifaceted campaigns which include a vaccinate-or-weara-mask-approach as well as mandatory declination policies. Policies, however, are often met by great opposition. In clinics where policies are infeasible, multifaceted campaigns comprised of extensive and individualized OSV and vaccination stands, a thorough educational and promotional campaign as well as incentives should be implemented to aim for an improved VC.

Overall, HCW influenza VC in Europe is far from satisfactory [4]. Although increasing the influenza $\mathrm{VC}$ in $\mathrm{HCW}$ remains a challenge, it is of utmost importance to protect our staff and our patients.

Author contributions SS designed the study, conceptualized and performed literature search, analyzed and interpreted the data, created the manuscript, created tables and figures, revised and approved the final manuscript. JSG created figures, revised and approved the final manuscript. OAC conceived the idea of the study and revised and approved the manuscript. SCM revised and approved the manuscript.

Funding Open Access funding enabled and organized by Projekt DEAL.

\section{Compliance with ethical standards}

Conflict of interest SS and JSG declare that they have no conflicts of interest. OAC is supported by the German Federal Ministry of Research and Education, is funded by the Deutsche Forschungsgemeinschaft (DFG, German Research Foundation) under Germany's Excellence Strategy-CECAD, EXC 2030-390661388 and has received research grants from Actelion, Amplyx, Astellas, Basilea, Cidara, Da Volterra, F2G, Gilead, Janssen, Medicines Company, Melinta, Merck/ MSD, Octapharma, Pfizer, Scynexis, is a consultant to Actelion, Allecra, Amplyx, Astellas, Basilea, Biosys, Cidara, Da Volterra, Entasis, F2G, Gilead, Matinas, MedPace, Menarini, Merck/MSD, Mylan, Nabriva, Noxxon, Octapharma, Paratek, Pfizer, PSI, Roche Diagnostics, Scynexis, and Shionogi, and received lecture honoraria from AlJazeera Pharmaceuticals, Astellas, Basilea, Gilead, Grupo Biotoscana, Merck/MSD and Pfizer. SCM was a consultant to Octapharma. She has been receiving research grants from the University Hospital of Cologne (KoelnFortune), from the German center for infection research (DZIF; Clinical Leave Stipend), and from the German Mycological Society (Dr. Manfred Plempel Stipend).

Open Access This article is licensed under a Creative Commons Attribution 4.0 International License, which permits use, sharing, adaptation, distribution and reproduction in any medium or format, as long as you give appropriate credit to the original author(s) and the source, provide a link to the Creative Commons licence, and indicate if changes were made. The images or other third party material in this article are included in the article's Creative Commons licence, unless indicated otherwise in a credit line to the material. If material is not included in the article's Creative Commons licence and your intended use is not permitted by statutory regulation or exceeds the permitted use, you will need to obtain permission directly from the copyright holder. To view a copy of this licence, visit http://creativecommons.org/licenses/by/4.0/.

\section{References}

1. Iuliano AD, et al. Estimates of global seasonal influenzaassociated respiratory mortality: a modelling study. Lancet. 2018;391:1285-300.

2. WHO. Influenza (Seasonal) Factsheet. [cited 2020 15.10.]. Available from https://www.who.int/news-room/fact-sheets/detail/influ enza-(seasonal).

3. Hayward AC. Influenza vaccination of healthcare workers is an important approach for reducing transmission of influenza from staff to vulnerable patients. PLoS ONE. 2017;12:e0169023.

4. ECDC, Seasonal influenza vaccination and antiviral use in EU/ EEA Member States-Overview of vaccine recommendation for 2017-2018 and vaccination coverage rates for 2015-2016 and 2016-2017 influenza seasons. 2018. 
5. Greenhawt M, Turner PJ, Kelso JM. Administration of influenza vaccines to egg allergic recipients: a practice parameter update 2017. Ann Allergy Asthma Immunol. 2018;120:49-52.

6. Neufeind J, et al. OKaPII-Studie zur Influenza-Impfung: Impfquoten und Impfmotivation bei Klinikpersonal in der InfluenzaSaison 2016/2017. Epid Bull. 2018;32:313-21.

7. Memoli MJ, et al. The natural history of influenza infection in the severely immunocompromised vs nonimmunocompromised hosts. Clin Infect Dis. 2014;58:214-24.

8. RKI. Ständige Impfkommission: Empfehlungen der Ständigen Impfkommission (STIKO) am Robert Koch-Institut. Epid Bull. 2019;34:313-64.

9. Wu D, et al. Coinfection of influenza virus and severe acute respiratory syndrome Coronavirus 2 (SARS-COV-2). Pediatr Infect Dis J. 2020;39:e79.

10. RKI (2020) Stellungnahme der Ständigen Impfkommision (STIKO) beim Robert Koch-Institut (RKI)-Bestätigung der aktuellen Empfehlungen zur saisonalen Influenzaimpfung für die Influenzasaison 2020/21 in Anbetracht der Auswirkung der COVID-19-Pandemie. Epid Bull 32/33: 28-30.

11. Podczervinski $\mathrm{S}$, et al. Employee influenza vaccination in a large cancer center with high baseline compliance rates: comparison of carrot versus stick approaches. Am J Infect Control. 2015;43:228-33

12. Abramson $\mathrm{ZH}$, et al. Randomized trial of a program to increase staff influenza vaccination in primary care clinics. Ann Fam Med. 2010;8:293-8.

13. Borgey F, et al. Effectiveness of an intervention campaign on influenza vaccination of professionals in nursing homes: a clusterrandomized controlled trial. Vaccine. 2019;37:1260-5.

14. Costantino $\mathrm{C}$, et al. Effectiveness of an educational intervention on seasonal influenza vaccination campaign adherence among healthcare workers of the Palermo University Hospital Italy. Ann Ig. 2019;31:35-44.

15. Cozza V, et al. Promotion of influenza vaccination among health care workers: findings from a tertiary care children's hospital in Italy. BMC Public Health. 2015;15:697.

16. Jung Y, Kwon M, Song J. Stepwise intervention including 1-on-1 counseling is highly effective in increasing influenza vaccination among health care workers. Am J Infect Control. 2017;45:635-41.

17. Llupia A, et al. New interventions to increase influenza vaccination rates in health care workers. Am J Infect Control. 2010;38:476-81.

18. Drees $\mathrm{M}$, et al. Carrots and sticks: achieving high healthcare personnel influenza vaccination rates without a mandate. Infect Control Hosp Epidemiol. 2015;36:717-24.

19. Guanche Gacell H, et al. A successful strategy for improving the influenza immunization rates of health care workers without a mandatory policy. Int J Occup Environ Med. 2015;6:184-6.

20. Horst-Schaper G et al (2019) Influenzaimpfung des medizinischen Personals: Klinikinterne Aktion "Be a flu fighter" schafft Trendwende. Dtsch Arztbl.

21. Marshall C, et al. Sustained improvement in staff influenza vaccination rates over six years without a mandatory policy. Infect Control Hosp Epidemiol. 2019;40:389-90.

22. Quan K, et al. Voluntary to mandatory: evolution of strategies and attitudes toward influenza vaccination of healthcare personnel. Infect Control Hosp Epidemiol. 2012;33:63-70.

23. Barbara A, et al. A campaign aimed at increasing seasonal influenza vaccination coverage among post graduate medical residents in an Italian teaching hospital. Hum Vaccin Immunother. 2019;15:967-72

24. Esolen LM, Kilheeney KL. Sustaining high influenza vaccination compliance with a mandatory masking program. Infect Control Hosp Epidemiol. 2014;35:603-4.
25. Oguz MM. Improving influenza vaccination uptake among healthcare workers by on-site influenza vaccination campaign in a tertiary children hospital. Hum Vaccin Immunother. 2019;15:1060-5.

26. Vimercati L, et al. Influenza vaccination in health-care workers: an evaluation of an on-site vaccination strategy to increase vaccination uptake in HCWs of a South Italy Hospital. Hum Vaccin Immunother. 2019;15:2927-32.

27. Gilardi $F$ et al (2018) Seasonal influenza vaccination in health care workers. A pre-post intervention study in an Italian Paediatric Hospital. Int J Environ Res Public Health 15.

28. Batabyal RA, et al. Impact of New York state influenza mandate on influenza-like illness, acute respiratory illness, and confirmed influenza in healthcare personnel. Infect Control Hosp Epidemiol. 2017:38:1361-3.

29. Huynh S, et al. Mandatory influenza vaccination of health care workers: a first-year success implementation by a community health care system. Am J Infect Control. 2012;40:771-3.

30. Rakita RM, et al. Mandatory influenza vaccination of healthcare workers: a 5-year study. Infect Control Hosp Epidemiol. 2010;31:881-8.

31. Awali RA, et al. Understanding health care personnel's attitudes toward mandatory influenza vaccination. Am J Infect Control. 2014;42:649-52.

32. Babcock HM, et al. Mandatory influenza vaccination of health care workers: translating policy to practice. Clin Infect Dis. 2010;50:459-64.

33. Smith DR, Van Cleave B. Influenza vaccination as a condition of employment for a large regional health care system. WMJ. 2012;111:68-71.

34. Kim H, et al. Evaluation of the impact of the 2012 Rhode Island health care worker influenza vaccination regulations: implementation process and vaccination coverage. J Public Health Manag Pract. 2015;21:E1-9.

35. Ksienski DS. Mandatory seasonal influenza vaccination or masking of British Columbia health care workers: Year 1. Can J Public Health. 2014;105:e312-6.

36. Modak RM, et al. Increasing influenza vaccination rates among hospital employees without a mandatory policy. Infect Control Hosp Epidemiol. 2012;33:1288-9.

37. Stuart RL, Gillespie EE, Kerr PG. A pilot study of an influenza vaccination or mask mandate in an Australian tertiary health service. Med J Aust. 2014;200:83-4.

38. Honda $\mathrm{H}$, et al. A successful strategy for increasing the influenza vaccination rate of healthcare workers without a mandatory policy outside of the United States: a multifaceted intervention in a Japanese tertiary care center. Infect Control Hosp Epidemiol. 2013;34:1194-200.

39. LaVela SL, et al. Healthcare worker influenza declination form program. Am J Infect Control. 2015;43:624-8.

40. Heinrich-Morrison K, et al. An effective strategy for influenza vaccination of healthcare workers in Australia: experience at a large health service without a mandatory policy. BMC Infect Dis. 2015;15:42.

41. Friedl A, et al. An intensive 5-year-long influenza vaccination campaign is effective among doctors but not nurses. Infection. 2012;40:57-62.

42. Frisina PG, et al. Increasing influenza immunization rates among healthcare providers in an ambulatory-based, University Healthcare Setting. Int J Qual Health Care. 2019;31:698-703.

43. Lytras $\mathrm{T}$, et al. Interventions to increase seasonal influenza vaccine coverage in healthcare workers: a systematic review and metaregression analysis. Hum Vaccin Immunother. 2016;12:671-81.

44. Lam PP, et al. Seasonal influenza vaccination campaigns for health care personnel: systematic review. CMAJ. 2010;182:E542-8. 
45. Hollmeyer $\mathrm{H}$, et al. Review: interventions to increase influenza vaccination among healthcare workers in hospitals. Influenza Other Respir Viruses. 2013;7:604-21.
46. Dorribo V, et al. Health care workers' influenza vaccination: motivations and mandatory mask policy. Occup Med (Lond). 2015;65:739-45. 